\title{
Propiedades psicométricas del Instrumento para evaluar capital psicológico en las Organizaciones Ipsicap-24*
}

\section{Psychometric Properties of the Instrument to Assess Psychological Capital in Organizations IPSICAP-24}

Recepción: 24 Octubre 2018 | Aceptación: 11 Octubre 2019

\author{
Leonor Emilia Delgado-Abella ${ }^{\mathrm{a}}$ \\ Universidad El Bosque, Colombia \\ ORCID: http://orcid.org/0000-0002-1591-2729 \\ Miguel Ángel Mañas Rodríguez \\ Universidad de Almería, España \\ ORCID: http://orcid.org/0000-0001-9771-0663
}

a Autor de correspondencia. Correo electrónico: leopsicopos@gmail.com

Para citar este artículo: Delgado-Abella, L. E., \& Mañas Rodríguez, M. Á. (2019). Propiedades psicométricas del Instrumento para evaluar capital psicológico en las Organizaciones Ipsicap-24. Universitas Psychologica, 18(5), 1-15. https://doi.org/10.11144/Javeriana.upsy 18-5.ppie

\section{RESUMEN}

La literatura científica ha reportado resultados importantes sobre el papel del capital psicológico como antecedente, consecuente, mediador o moderador de variables del comportamiento organizacional. Partiendo de la necesidad de instrumentos de medida adecuados para cada población, el objetivo de esta investigación es presentar un análisis de las propiedades psicométricas del Ipsicap-24. En el estudio participaron tres muestras de trabajadores colombianos provenientes de organizaciones de varios sectores de la economía y caracterización sociodemográfica variada. Con datos de la primera muestra $(N=1517)$, dividida aleatoriamente en dos partes, se realizaron Análisis Factorial Exploratorio y Confirmatorio, así el Ipsicap original de 36 ítems dio origen al Ipsicap-24, conformado por cuatro escalas: esperanza (7 ítems), optimismo ( 7 ítems), resiliencia (6 ítems) y autoeficacia (4 ítems). Para obtener evidencia de validez convergente y divergente del Ipsicap-24, respectivamente, la segunda muestra $(N=428)$ respondió el Cuestionario Utrecht Work Engagement (UWES); y la tercera muestra $(N=105)$, el Cuestionario General de Salud (General Health Questionnaire, GHQ-28). Los resultados obtenidos aportan evidencia de consistencia interna y validez de constructo para el Ipsicap-24. Esta nueva herramienta permite la evaluación válida y confiable del capital psicológico en trabajadores colombianos.

\section{Palabras clave}

capital psicológico; instrumentos de evaluación; propiedades psicométricas; psicología organizacional positiva; comportamiento organizacional positivo.

\begin{abstract}
The scientific literature has reported important results on the role of psychological capital as antecedent, consequent, mediator or moderator of organizational behavior variables. Based on the need for adequate measurements for each population, the objective of this research is to present an analysis of the psychometric properties of IPSICAP-24. Three samples of Colombian workers from organizations in various sectors of the economy and varied sociodemographic characterization participated
\end{abstract}


in the study. With data from the first sample $(\mathrm{N}=$ 1517), randomly divided into two parts, Exploratory and Confirmatory Factor Analysis were performed, thus the original IPSICAP of 36 items gave rise to IPSICAP-24, consisting of four scales: hope (7 items) , optimism (7 items), resilience (6 items) and self-efficacy (4 items). In order to obtain evidence of convergent and divergent validity of IPSICAP-24, respectively, the second sample $(N=428)$ answered the Utrecht Work Engagement Questionnaire (UWES); and the third sample $(N=105)$, the General Health Questionnaire (GHQ-28). The results obtained provide evidence of internal consistency and construct validity for IPSICAP-24. This new tool allows the valid and reliable evaluation of psychological capital in Colombian workers.

Keywords

psychological capital; psychological measurements; psychometric properties; positive organizational psychology; positive organizational behavior.

Siguiendo a Luthans, Youssef y Avolio (2007), el capital psicológico se puede definir como un estado de desarrollo positivo que permite a la persona 1) tener confianza para realizar los esfuerzos que sean necesarios con el fin de tener éxito en tareas retadoras (auto-eficacia), 2) hacer atribuciones de causalidad positivas acerca de los sucesos presentes y futuros (optimismo), 3) perseverar en el logro de los objetivos y, siempre que sea necesario, redireccionar las estrategias para alcanzarlos de manera exitosa (esperanza), y 4) ante los problemas y la adversidad, mantenerse en pie, volver a comenzar e ir más allá para lograr el éxito (resiliencia).

En el contexto organizacional, el capital psicológico como constructo de segundo orden se ha caracterizado por aportar mayor explicación de la varianza, que la que podrían generar sus cuatro componentes de forma separada de variables como: desempeño y satisfacción (Luthans, Avolio, Avey, \& Norman, 2007), la conducta de compartir conocimiento (DelgadoAbella \& Castañeda Zapata, 2011), y síntomas de depresión (Liu, Hu, Wang, Sui, \& Ma, 2013).

Gracias a sus comunalidades, la esperanza, el optimismo, la resiliencia y la autoeficacia actúan de forma complementaria para crear una sinergia (Dawkins, Martin, Scott, \& Sanderson, 2013) que dota a la persona de una sensación de control, intencionalidad y capacidad para la búsqueda activa de sus objetivos. Estos cuatro componentes del constructo, por separado y en conjunto, articulan mecanismos emocionales, cognitivos, motivacionales y sociales (Luthans $\&$ Youssef-Morgan, 2017), que se acoplan para impactar positivamente el desempeño de los trabajadores y les permite contar con una "evaluación positiva de las circunstancias y de la probabilidad de éxito basados en motivación, esfuerzo y perseverancia" (Luthans et al., 2007, p. 550).

Aunque estas cuatro variables son estados psicológicos positivos (Avey, Luthans, \& Youssef, 2010) y, en consecuencia, son susceptibles de entrenamiento y desarrollo, es claro que guardan diferencias importantes entre sí. En primer lugar, se pueden conceptualizar psicológicamente así: la autoeficacia, como una creencia; el optimismo, como estilo atribucional; la esperanza, como estado motivacional y la resiliencia, como capacidad (Delgado-Abella, 2013).

En casi tres lustros de investigación sobre capital psicológico se encuentra nutrida evidencia de su influencia sobre variables de resultado a nivel individual, grupal y organizacional; y también, aunque en menor medida, de su impacto como antecedente mediador o moderador de variables del comportamiento organizacional (Newman, Ucbasaran, Zhu, \& Hirst, 2014; Srivastava \& Maurya, 2017; Luthans \& Youssef-Morgan, 2017; Nolzen, 2018). En general, el capital psicológico se relaciona de forma positiva con actitudes y conductas deseables de los empleados, y de forma negativa con variables no deseadas en el contexto organizacional (Avey, Reichard, Luthans \& Mhatre, 2011). Adicionalmente se han reportado en la literatura algunos estudios sobre capital psicológico como un fenómeno del nivel de los equipos, es decir, capital psicológico colectivo (Rego et al., 2019; Somech, \& Khotaba, 2017; Heled, Somech, \& Waters, 2016; Walumbwa, Luthans, Avey, \& Oke, 2011; Clapp-Smith, Vogelgesang, \& Avey, 2009).

La primera medida del capital psicológico fue desarrollada por Luthans et al. (2007), y es conocida como PCQ, por su sigla en inglés derivada de Psychological Capital Questionnaire. 
Se trata de un cuestionario de 24 ítems, 6 para cada uno de los componentes del capital psicológico, en escala tipo Likert con seis opciones de respuesta para identificar el grado de acuerdo de cada persona con cada afirmación en el momento de resolver la prueba.

El PCQ-24 ha sido adaptado a la población de Colombia (Delgado-Abella \& Castañeda, 2011), Sudáfrica (Görgens-Ekermans \& Herbert, 2013), Tailandia (Sapyaprapa, Tuicomepee, \& Watakakosol, 2013), España (Azanza, Domínguez, Moriano, \& Molero, 2014), Italia (Alessandri, Borgogni, Consiglio, \& Mitidieri, 2015), Portugal (Antunes, Caetano, \& Pina e Cunha, 2017) y Rumania (Lupşa \& Vîrgă, 2018).

La versión de 12 ítems, el PCQ-12, fue ajustada para población española por León-Pérez, Antino y León-Rubio (2017) y por Djourova, Rodríguez y Lorente-Prieto (2019). Otros autores han publicado análisis de propiedades psicométricas del PCQ-12 en varios países, a saber: Brasil (Kamei et al., 2018), México (Santana-Cárdenas, Viseu, López-Núñez, \& Jesus, 2018) y España (López-Núñez, de Jesús, Viseu, \& Santana-Cárdenas, 2018).

A partir del PCQ se han desarrollado otras medidas del capital psicológico: capital psicológico transcultural (Dollwet \& Reichard, 2014). Por su parte, Harms y Luthans (2012) propusieron un instrumento basado en una técnica semiproyectiva para evaluar el capital psicológico implícito, denominado Implicit Psychological Capital Questionnaire, IPCQ. Posteriormente, con base en el I-PCQ, se diseñó el Implicit Psychological Capital Health Measure (IPCQ-H), con el fin de identificar esquemas implícitos o cogniciones acerca de la salud de las personas (Harms, Vanhove, \& Luthans, 2017).

Desde otra perspectiva, Omar, Salessi y Urteaga (2014), en concordancia con las recomendaciones de especialistas acerca de "disponer de medidas que reflejen la idiosincrasia del lugar donde se las aplique" (p. 316), diseñaron y validaron la escala Cappsi para medir capital psicológico en Argentina, lo que constituye un valioso aporte para la investigación y también para diagnóstico e intervención en el terreno organizacional. En esta misma dirección, Lorenz, Beer, Pütz y Heinitz (2016) presentan el Compound PsyCap Scale (CPC-12) en Alemania, como una medida útil para investigación en varios campos, y no solamente para población trabajadora. En España, Meseguer-de Pedro, Soler-Sánchez, Fernández-Valera y García-Izquierdo (2017) crearon el cuestionario OREA (optimismo, resiliencia, esperanza y autoeficacia) para evaluar el capital psicológico en el ámbito laboral; a modo de justificación para la elaboración de una nueva escala, los autores señalan que en términos de validez de contenido, algunos ítems del PCQ están lejos del promedio de la realidad de los trabajadores por no corresponder a las actividades y tareas que muchos de ellos deben ejecutar, citan a modo de ejemplo los siguientes ítems: "confío en mí mismo cuando represento a mi departamento en reuniones con la gerencia", "me siento seguro al presentar nueva información a un grupo de pares" y "puedo contribuir en las discusiones estratégicas sobre la organización".

En Colombia, como se mencionó anteriormente, en el marco de una investigación sobre la conducta de compartir conocimiento y su relación con el capital psicológico, DelgadoAbella y Castañeda Zapata (2011) realizaron la adaptación del PCQ. A partir de esa experiencia, se encontró evidencia compatible con lo señalado en las líneas precedentes, en torno a temas de validez de contenido del PCQ en esta región del mundo: primero, es necesario contar con instrumentos de evaluación culturalmente compatibles con el lugar de aplicación de estos; $y$, segundo, el contenido de algunos ítems de la prueba va dirigido solo a un sector de la población trabajadora. Adicionalmente, el comportamiento de los datos mostró cierto efecto de techo, es decir, la mayoría de los participantes obtuvo puntuaciones muy altas del constructo. Una posible explicación para este hecho es que el significado y alcance de sus cuatro variables constitutivas, en un país con las condiciones sociopolíticas de Colombia, puede ser cualitativamente diferente, es decir, principalmente el optimismo, la resiliencia y la esperanza pueden haber adquirido un rango "más 
amplio" por la sobreexposición a la violencia y la creciente necesidad de afrontamiento. Estas consideraciones dieron origen a la creación del Ipsicap.

La construcción del Ipsicap tuvo lugar en 2011, sus autoras son las psicólogas Leonor Delgado-Abella, Luisa Devia y Carolina Martínez. Con base en una revisión de la literatura se planteó la estructura de prueba atendiendo a la conceptualización psicológica de cada una de las cuatro variables, se realizó el proceso de validación de contenido mediante el Método Angoff Modificado y el análisis de los resultados del estudio piloto de la prueba desde el modelo de Rasch (DelgadoAbella, Devia, \& Martínez, 2018; DelgadoAbella, 2013). El uso del método Angoff (Ricker, 2006) permitió contar simultáneamente con nueve jurados para conceptuar sobre la estructura general de la prueba y sobre cada uno de los ítems; el grupo de pares estuvo conformado por expertos en psicología organizacional, psicología positiva, psicometría y gerencia de talento humano. Adicionalmente, se realizaron entrevistas cognitivas con trabajadores para verificar la comprensión de cada ítem.

El objetivo de este artículo es presentar el análisis de las propiedades psicométricas del Ipsicap a partir de estudios realizados con tres muestras de trabajadores colombianos. Específicamente, se busca poner a prueba la consistencia interna y validez de constructo del Ipsicap y, mediante la aplicación del UWES y el GHQ-28, obtener evidencia de su validez convergente y divergente, respectivamente.

\section{Método}

\section{Participantes}

Para esta investigación se contó con tres muestras seleccionadas por conveniencia. La primera, integrada por 1517 personas de varias organizaciones domiciliadas en la ciudad de Bogotá, y pertenecientes a varios sectores económicos. El 48.35 \% fueron mujeres y el $51.43 \%$ fueron hombres. La edad promedio fue de 33.13 años y el grupo etario con mayor representación $(27.7 \%)$ estuvo ubicado en el rango de 18 a 25 años, seguido por el grupo entre 26 y 30 años (19.1\%) y las personas entre 31 y 35 años (15.21\%). En cuanto al nivel de escolaridad, el $19.1 \%$ tiene formación técnica o tecnológica, y el 39.6 $\%$ es profesional universitario. Sobre el tipo de cargo, $34.02 \%$ se desempeña ocupando cargos operativos (operarios, ayudantes, servicios generales, vigilancia), el $18 \%$ en cargos auxiliares (auxiliar, asistente, cajero, supervisor operativo), y un $39.38 \%$ en cargos profesionales.

Con el fin de obtener evidencia de validez convergente y divergente, se trabajó con otras dos muestras. La segunda, compuesta por 428 trabajadores de organizaciones públicas y privadas de la ciudad de Bogotá, cuya edad promedio fue de 35.6 años. La tercera muestra, de 105 empleados de un colegio privado de Bogotá, con una edad promedio de 38.8 años. La información sobre la segunda y tercera muestra se consolida en la Tabla 1.

\section{Tabla 1}

Información sociodemográfica de las muestras 2 y 3 en porcentaje

\begin{tabular}{|c|c|c|c|c|c|c|c|}
\hline \multicolumn{2}{|c|}{$\begin{array}{c}\text { Variables } \\
\text { sociodemograficas }\end{array}$} & \multirow{3}{*}{$\begin{array}{c}\begin{array}{c}\text { Muestra 2 } \\
N=428\end{array} \\
52.8 \\
7\end{array}$} & \multirow{2}{*}{$\begin{array}{r}\begin{array}{c}\text { Muestra 3 } \\
N=105\end{array} \\
80\end{array}$} & \multicolumn{2}{|c|}{$\begin{array}{c}\text { Variable } \\
\text { sociodemográficas }\end{array}$} & \multirow{2}{*}{$\begin{array}{l}\begin{array}{l}\text { Muestra 2 } \\
N=428\end{array} \\
17.3\end{array}$} & \multirow{2}{*}{$\begin{array}{r}\begin{array}{r}\text { Muestra 3 } \\
N=105\end{array} \\
3.81\end{array}$} \\
\hline \multirow{4}{*}{ Sexo } & & & & & Secundaria & & \\
\hline & Hombres & & 20 & & Técnicos & 17.3 & 13.33 \\
\hline & 18 a 30 años & 35.1 & 17.1 & & Tecnólogos & 6.8 & 5.71 \\
\hline & 31 a 40 años & 37.4 & 40 & Escolaridad & Profesionales & 43.9 & 60 \\
\hline \multirow[t]{4}{*}{ Edad } & 41 a 50 años & 18 & 32.4 & & Especialización & 8.4 & 11.4 \\
\hline & 51 a 60 años & 8.2 & 8.6 & & Maestria & 4.9 & 5.7 \\
\hline & 61 a 80 años & 1.4 & 1.9 & & Doctorado & 1.4 & 0 \\
\hline & Casados & 32.7 & 41.9 & & Operativos & 6.8 & 0 \\
\hline \multirow{4}{*}{$\begin{array}{l}\text { Estado } \\
\text { civil }\end{array}$} & Separados & 3.3 & 3.8 & & Auxiliar & 24.5 & 11.43 \\
\hline & Solteros & 42.1 & 36.1 & Cargo & Profesionales & 55.1 & 77.1 \\
\hline & Viudos & 0.9 & 2.9 & & Jefatura & 7.7 & 9.52 \\
\hline & T. fijo & 48.4 & 90.5 & & Gerencia & 3.5 & 1.9 \\
\hline \multirow{3}{*}{$\begin{array}{l}\text { Tipo de } \\
\text { contrato }\end{array}$} & T. indefinido & 30.4 & 9.5 & & & & \\
\hline & Temporales & 2.6 & 0 & & & & \\
\hline & Honorarios & 18.7 & 0 & & & & \\
\hline
\end{tabular}

\section{Instrumentos}

Instrumento para evaluar capital psicológico en las organizaciones (Ipsicap)

Consta de 36 ítems en escala Likert de seis puntos, así: completamente en desacuerdo 1), en desacuerdo 2), algo en desacuerdo 3), algo de acuerdo 4), de acuerdo 5) y completamente de acuerdo 6). Se evalúan las cuatro variables constitutivas del constructo capital psicológico, definidas de la siguiente 
forma: "esperanza: perseverancia para alcanzar las metas y la capacidad para establecer varios caminos que conduzcan a su logro de forma exitosa; optimismo: tendencia a hacer atribuciones de causalidad externas y temporales a los acontecimientos negativos y estables e internas para experiencias positivas; resiliencia: capacidad para adaptarse a los cambios propios de la vida organizacional, reponerse a los fracasos y superar los estados en los que estaba inicialmente; y autoeficacia: convicción personal de contar con las habilidades necesarias para alcanzar las metas que le permiten asumir tareas retadoras" (Delgado-Abella et al., 2018).

\section{Cuestionario Utrecht Work Engagement (UWES)}

Elaborado por Schaufeli y Bakker (2003), traducido al español y adaptado lingüística y culturalmente para la población colombiana por Ospina y Delgado-Abello (2014). Consta de 17 ítems que miden las tres variables del constructo engagement: vigor, con seis ítems; dedicación con cinco ítems; y absorción con seis ítems. La escala de respuesta de siete puntos va desde nunca hasta siempre. El coeficiente Alfa de Cronbach obtenido con la segunda muestra de esta investigación es 0.911 .

\section{Cuestionario General de Salud (General Health Questionnaire, GHQ-28)}

Este instrumento posee cuatro subescalas: Síntomas Psicosomáticos, Ansiedad y Problemas de Sueño, Depresión y Disfunción Social; cada una con siete ítems tipo Likert que van desde $1=$ no, en lo absoluto, a $4=$ más de lo habitual (Goldberg \& Hillier, 1979). Con la tercera muestra utilizada para el presente estudio se obtuvo un Alfa de Cronbach de 0.891.

\section{Procedimiento}

El Ipsicap se aplicó a 1517 trabajadores de organizaciones colombianas (muestra 1), previa obtención de los permisos necesarios de sus directivos responsables y la firma del consentimiento informado de cada uno de los participantes, quienes respondieron el cuestionario voluntariamente. El instrumento fue aplicado por profesionales de la psicología en cada una de las organizaciones.

Después del proceso de depuración total del Ipsicap, se obtuvieron medidas con dos muestras adicionales para encontrar evidencia de validez convergente y divergente. En el primer caso, se aplicó el Ipsicap depurado con 24 ítems (a partir de ahora denominado Ipsicap-24) y el UWES a una muestra de 428 colaboradores de varias empresas (muestra 2); y en el segundo caso, la muestra estuvo conformada por 105 empleados administrativos y docentes de una institución educativa de enseñanza preescolar, básica y media (muestra 3), quienes respondieron el Ipsicap-24 y el GHQ-28. En ambos casos, las organizaciones están ubicadas en la ciudad de Bogotá.

\section{Análisis de datos}

El tratamiento estadístico de los datos se llevó a cabo mediante IBM SPSS 23. En primer lugar, con la muestra 1 se realizó un procedimiento de imputación lineal múltiple para la corrección de datos ausentes (missing), la identificación de casos atípicos (outliers) y la prueba de distancia de Mahalanobis. Como resultado se obtuvo una reducción de la muestra a 1385 personas. Posteriormente, utilizando un método aleatorio se hizo una partición de la muestra, la cual quedó dividida en dos partes: una de 683 y la otra de 702 individuos. La primera se usó para el Análisis Factorial Exploratorio (AFE) y la segunda para el Análisis Factorial Confirmatorio (AFC).

Para verificar la suficiencia y pertinencia de las dos muestras para el AFE y el AFC se usaron el coeficiente Kaiser-Meyer-Olkin (KMO) y la prueba de esfericidad de Bartlett (Tabla 2). Estos indicadores reflejan que las dos muestras son aptas para la realización de los análisis factoriales previstos, en cada caso. 
Tabla 2

Coeficiente KMO y prueba de esfericidad de

Bartlett para las dos muestras

\begin{tabular}{cccccc}
\hline Muestra & $N$ & $K M O$ & $\chi^{2}$ & $g l$ & $P$ \\
\hline AFE & 683 & 0.926 & 4838.024 & 278 & 0 \\
AFC & 702 & 0.881 & 11829.688 & 276 & 0 \\
\hline
\end{tabular}

\section{Resultados}

En primer lugar, como un indicador de la medida de fiabilidad del test, se calculó el coeficiente Alfa de Cronbach para cada escala y el total del Ipsicap-24, como se ilustra en la tabla 3. De acuerdo con Tavakol y Dennick (2011), los coeficientes obtenidos cada una de las escalas y para el Ipsicap-24 son adecuados.

\section{Tabla 3}

Medidas descriptivas y de fiabilidad para el

Ipsicap-24

\begin{tabular}{lrrrrr}
\hline \multicolumn{1}{c}{ Estadístico } & Esperanza & Optimismo & Resiliencia & Autoeficacia & Ipsicap-24 \\
\hline Alfa de Cronbach & 0.84 & 0.78 & 0.83 & 0.71 & 0.93 \\
Media & 35.89 & 33.61 & 28.58 & 18.44 & 116.52 \\
Desviación Estándar & 4.51 & 3.95 & 3.95 & 2.72 & 12.14 \\
Varianza & 20.31 & 15.63 & 15.63 & 7.38 & 147.4 \\
Mínimo & 7 & 9 & 6 & 4 & 35 \\
Máximo & 42 & 42 & 36 & 24 & 141 \\
Número de ítems & 7 & 7 & 6 & 4 & 24 \\
\hline
\end{tabular}

Otra medida de consistencia interna de la prueba está dada por las correlaciones entre sus escalas (Cortina, 1993; Tang, Cui, \& Babenko, 2014). En las Tablas 6 y 7 se exhiben las matrices de correlación entre las cuatro escalas y el Ipsicap-24 completo, obtenidas con las muestras 2 y 3 del presente estudio, respectivamente. En ambos casos, todas las correlaciones son directas y significativas $(p<0.01)$, lo cual permite establecer que la prueba posee un nivel adecuado de consistencia interna. Para la segunda muestra $(N=428)$, las correlaciones entre las binas formadas por esperanza, optimismo y resiliencia oscilan entre $r=0.67$ y $r=0.716$; las binas entre autoeficacia y los otros tres componentes del constructo se ubican entre $r=0.530$ y $r$ $=0.597$; y la correlación entre las escalas y el total de la prueba van entre $r=0.75$ y $r=$ 0.892. Con respecto a la tercera muestra $(N$ $=105)$, el comportamiento de las correlaciones es similar, aunque con coeficientes un poco más bajos: las binas formadas por esperanza, optimismo y resiliencia oscilan entre $r=0.515$ y $r=0.597$; las binas entre autoeficacia y los otros tres componentes del constructo se ubican entre $r=0.336$ y $r=0.445$; y la correlación entre las escalas y el total de la prueba van entre $r=0.645$ y $r=0.838$.

Por otra parte, para estimar la validez de Constructo se ejecutó primero un Análisis Factorial Exploratorio y un Análisis Factorial Confirmatorio. Estos procesamientos se realizaron con la primera muestra $(N=1385)$. Es pertinente recordar que aleatoriamente esta muestra quedó dividida en dos partes: una de 683 y la otra de 702 individuos. La primera se usó para el AFE y la segunda para el AFC.

A partir de los resultados iniciales del AFE, de los 36 ítems originales del Ipsicap se tomó la decisión de eliminar de la prueba los inversos: $3,4,12,24,26,29,30,32,35$ y 36. De igual forma, por covariar de manera negativa, se eliminaron los ítems 17 y 22. Con los 24 ítems restantes, se hicieron procesamientos de análisis de componentes principales con método de rotación Oblimin y normalización Kaiser, de cuatro factores fijos, que explicaron el 46.12 $\%$ de la varianza total. La estructura factorial resultante se muestra en la Tabla 4. 


\section{Tabla 4}

Estructura factorial de cada una de las dimensiones del Ipsicap-24

\begin{tabular}{|c|c|c|c|c|c|}
\hline \multirow{2}{*}{ ftem } & \multirow{2}{*}{$\begin{array}{c}\text { Escala } \\
\text { Original }\end{array}$} & \multicolumn{4}{|c|}{ Componente } \\
\hline & & & & 3 & 4 \\
\hline 1. Me mantengo firme en la lucha por alcanzar mis metas & & 0.749 & 0.017 & -0.046 & 0.018 \\
\hline 2. Considero los obstáculos como retos que debo superar & Esperanza & 0.72 & 0.151 & -0.058 & 0.025 \\
\hline $\begin{array}{l}\text { 3. Establezco con facilidad las rutas que me sirven para } \\
\text { lograr los objetivos }\end{array}$ & Esperanza & 0.427 & -0.203 & 0.280 & 0.002 \\
\hline 4. Identifico estrategias para alcanzar mis metas & Esperanza & 0.614 & -0.118 & 0.182 & 0.011 \\
\hline $\begin{array}{l}\text { 5. Cuando un plan me falla busco otras opciones que } \\
\text { funcionen para cumplir con el objetivo }\end{array}$ & Esperanza & 0.696 & -0.045 & 0.032 & 0.008 \\
\hline 6. Me resulta dificil abandonar mis metas & Esperanza & 0.505 & 0.186 & -0.102 & -0.158 \\
\hline $\begin{array}{l}\text { 7. Para lograr lo que he planeado me esfuerzo por } \\
\text { implementar estrategias que conduzcan a su logro }\end{array}$ & Esperanza & 0.674 & -0.119 & 0.119 & 0.044 \\
\hline $\begin{array}{l}\text { 8. Creo que los objetivos alcanzados en el trabajo se } \\
\text { deben a mi buen desempeño }\end{array}$ & Optimismo & 0.747 & -0.022 & 0.061 & -0.056 \\
\hline $\begin{array}{l}\text { 9. Pienso que el cargo que ocupo en la empresa me to he } \\
\text { ganado con esfuerzo y dedicación }\end{array}$ & Optimismo & 0.657 & -0.165 & -0.007 & 0.149 \\
\hline $\begin{array}{l}\text { 10. Con el trabajo que tengo puedo alcanzar las metas que } \\
\text { he proyectado en mi vida }\end{array}$ & Optimismo & 0.054 & -0.223 & 0.265 & 0.541 \\
\hline $\begin{array}{l}\text { 11.Aun en momentos de dificultad creo que es posible un } \\
\text { futuro mejor }\end{array}$ & Optimismo & 0.445 & 0.183 & 0.053 & 0.219 \\
\hline $\begin{array}{l}\text { 12. Existen diferentes situaciones que me facilitan el logro } \\
\text { de una meta }\end{array}$ & Optimismo & 0.230 & 0.031 & 0.187 & 0.375 \\
\hline $\begin{array}{l}\text { 13.Cuando se obtienen resultados negativos en el trabajo } \\
\text { suelo pensar en lo que pude haber hecho para evitarlo }\end{array}$ & Optimismo & 0.073 & 0.391 & 0.055 & 0.333 \\
\hline $\begin{array}{l}\text { 14. Cuando algo bueno me ocurre en el trabajo creo que es } \\
\text { porque mis compañeros de trabajo me ayudan }\end{array}$ & Optimismo & -0.157 & 0.156 & -0.221 & 0.778 \\
\hline $\begin{array}{l}\text { 15. Las experiencias dificiles de mi vida me han llevado a } \\
\text { hacer cosas que no habia pensado ser capaz de realizar }\end{array}$ & Resiliencia & 0.005 & 0.684 & 0.095 & 0.103 \\
\hline $\begin{array}{l}\text { 16.El fracaso obtenido en la realización de una labor me } \\
\text { ha impulsado a insistir al punto de lograr sobrepasar lo } \\
\text { inicialmente esperado }\end{array}$ & Resiliencia & 0.441 & 0.463 & 0.014 & 0.114 \\
\hline 17.Aun con dificultad, he superado los eventos negativos & Resiliencia & 0.350 & 0.352 & 0.165 & 0.104 \\
\hline $\begin{array}{l}\text { 18. Cuando se presentan condiciones adversas en mi } \\
\text { trabajo, logro cumplir los objetivos propuestos } \\
\text { oportunamente }\end{array}$ & Resiliencia & 0.159 & 0.042 & 0.604 & 0.008 \\
\hline $\begin{array}{l}\text { 19. Cuando existen inconsistencias en las condiciones } \\
\text { laborales, logro cumplir con los objetivos propuestos }\end{array}$ & Resiliencia & -0.265 & 0.059 & 0.867 & -0.074 \\
\hline $\begin{array}{l}\text { 20.Mi desempeño laboral se mantiene estable, aun frente a } \\
\text { los cambios que surgen dentro de la empresa }\end{array}$ & Resiliencia & 0.158 & -0.015 & 0.558 & 0.191 \\
\hline 21.Me involu & Autoeficacia & 0.309 & 0.075 & 0.492 & -0.009 \\
\hline & Autoeficacia & 0.364 & 0.069 & 0.443 & -0.062 \\
\hline $\begin{array}{l}\text { 23.Me siento bien cuando realizo tareas dificiles } \\
\text { 24.Me incomoda encargarme de tareas demasiado fáciles }\end{array}$ & $\begin{array}{l}\text { Autoeficacia } \\
\text { Autoeficacia }\end{array}$ & $\begin{array}{r}0.326 \\
-0.095\end{array}$ & $\begin{array}{l}0.223 \\
0.351\end{array}$ & $\begin{array}{l}0.348 \\
0.019\end{array}$ & $\begin{array}{r}0.013 \\
-0.223\end{array}$ \\
\hline
\end{tabular}

Posteriormente, con la muestra conformada por 702 personas y con el Ipsicap-24, mediante AFC, se pusieron a prueba los siguientes modelos de medida:

a. Modelo de medida 1: pone a prueba el capital psicológico como constructo unidimensional, es decir, no considera los componentes.

b. Modelo de medida 2: a partir del Ipsicap original, representa la composición del constructo capital psicológico con sus cuatro variables constitutivas: esperanza, optimismo, resiliencia y autoeficacia.

c. Modelo de medida 3: producto del AFE realizado con los datos de la muestra 1 del presente estudio, de acuerdo con la estructura factorial obtenida (Tabla 4).

d. Modelo de medida 4: resultante del ajuste del modelo de medida 2, a partir de la información suministrada por los índices de modificación (Figura 1).
Tabla 5

Índices de ajuste de cada uno de los modelos de medida

\begin{tabular}{ccrrrrrrr}
\hline Modelo & CMIN & DF & CMIN/DF & GFI & TLI & CFI & RMSEA & \multicolumn{1}{c}{ AIC } \\
\hline 1 & 4986.64 & 252 & 19.788 & 0.563 & 0.557 & 0.595 & 0.164 & 5082.64 \\
2 & 777.86 & 247 & 3.149 & 0.906 & 0.872 & 0.885 & 0.056 & 883.86 \\
3 & 644.44 & 224 & 2.87 & 0.922 & 0.891 & 0.903 & 0.052 & 748.442 \\
4 & 461.81 & 230 & 2 & 0.95 & 0.94 & 0.95 & 0.038 & 601.817 \\
\hline \multicolumn{7}{c}{ Nota.Las estimaciones son } & & \\
& \multicolumn{8}{c}{ significativas a p < 0.001 }
\end{tabular}

Con base en los criterios de referencia y de bondad de ajuste propuestos por Ruiz, Pardo y San Martín (2010), se espera que la medida de ajuste absoluto expresada por la razón Chi-cuadrado/grados de libertad (CMIN/ $\mathrm{DF}$ ) sea menor que 3. Así entonces, en la Tabla 5 se aprecia que solo los modelos 3 y 4 cumplen con condición. El Índice de Bondad de ajuste comparativo (CFI $=\geq 0.95$, valor recomendado) únicamente resulta óptimo para el modelo 4 , situación que se ratifica mediante el Índice de Tucker-Lewis (TLI), por cuanto desde la perspectiva de Escobedo Portillo, Hernández Gómez, Estebané Ortega, y Martínez Moreno (2016) su valor mínimo aceptable es 0.9.

En la misma dirección, al considerar los criterios propuestos por Ruiz et al. (2010) con respecto al Índice de Bondad de ajuste (GFI $=\geq$ 0.95, valor recomendado), y a la Raíz del Residuo Cuadrático promedio (RMSEA $=<0.08$, valor recomendado), se puede establecer que el modelo 4 ha mostrado mayor bondad de ajuste.

En conclusión, los indicadores de ajuste obtenidos por los cuatro modelos (Tabla 5) muestran que el modelo 1 (unidimensional) no es viable. Los modelos 2 y 3 cumplen solo algunos de los criterios. El modelo 4, que conserva la estructura original del constructo capital psicológico, presenta un ajuste excelente y, además, obtuvo el valor más bajo en el Criterio de Información de Akaike (AIC) entre los cuatro modelos. Por tanto, se puede inferir que es el de mayor parsimonia, y por ser esta una medida comparativa, resulta ser el mejor modelo (Escobedo Portillo et al., 2016). 
Figura 1.

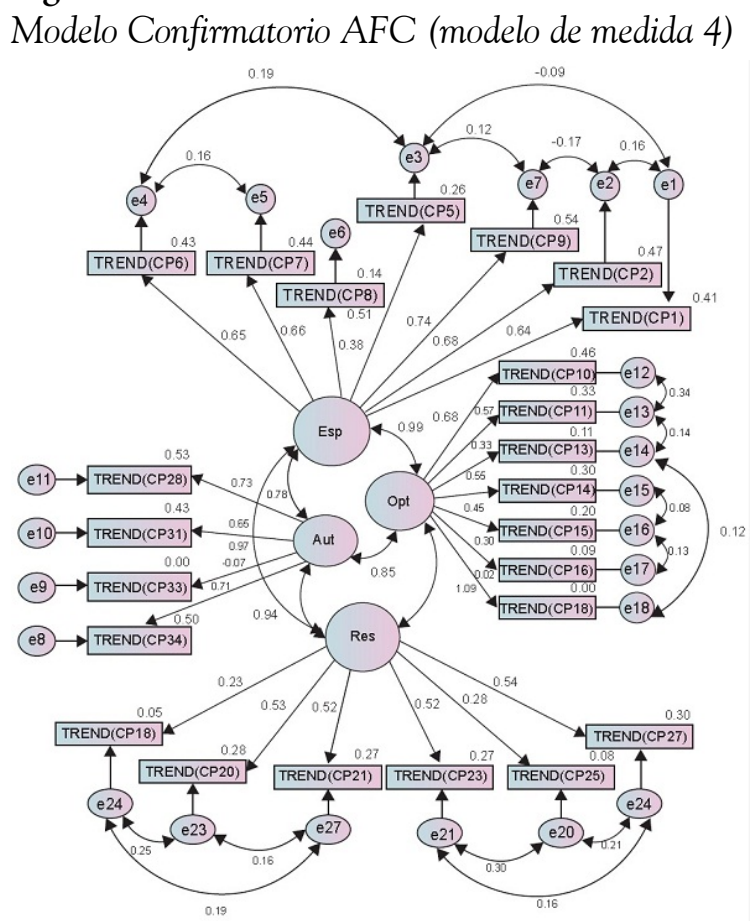

Adicionalmente, para obtener evidencia de validez convergente se trabajó con la segunda muestra de este estudio $(N=$ 428), estos trabajadores respondieron el Ipsicap-24 y el UWES adaptado para población colombiana. Esta última prueba mide engagement, variable en la literatura sobre comportamiento organizacional positivo, que ha mostrado estar relacionada positivamente con capital psicológico (Simons \& Buitendach, 2013; De Waal \& Pienaar, 2013; Görgens-Ekermans \& Herbert, 2013; Paek, Schuckert, Kim \& Lee, 2015; Karatepe \& Karadas, 2015; Lorenz et al., 2016; Li, Castaño, \& Li, 2018). Todas las correlaciones, entre las respectivas dimensiones, y capital psicológico y engagement, son positivas y significativas $(p<0.01)$, como se indica en la Tabla 6. Estas correlaciones oscilan entre $r$ $=0.379$ (dedicación-autoeficacia) y $r=0.66$ (Ipsicap total-UWES total), es decir, se ubican en un rango medio.
Tabla 6

Matriz de Correlaciones Ipsicap-24-UWES

\begin{tabular}{|c|c|c|c|c|c|c|c|c|}
\hline Constructo/Dimensión & 1 & 2 & 3 & 4 & 5 & 6 & 7 & 8 \\
\hline 1. UWES Vigor & & & & & & & & \\
\hline 2. UWES Dedicación & $\begin{array}{l}0.753^{* *} \\
0.756^{* *}\end{array}$ & $\stackrel{1}{1}$ & & & & & & \\
\hline $\begin{array}{l}\text { 3. UWES Absorción } \\
\text { 4. UWES Total }\end{array}$ & $\begin{array}{r}0.756 \\
0.92^{* *}\end{array}$ & $0.895^{* *}$ & $0.918^{* *}$ & 1 & & & & \\
\hline 5. Ipsicap Esperanza & $0.56^{* *}$ & $0.489^{* *}$ & $0.486^{* *}$ & $0.562^{* *}$ & 1 & & & \\
\hline 6. Ipsicap Optimismo & $0.552^{* *}$ & $0.57^{*+}$ & $0.533^{* *}$ & $0.604^{* *}$ & $0.67^{* *}$ & 1 & & \\
\hline 7. Ipsicap Resiliencia & $0.587^{* *}$ & $0.54^{* *}$ & $0.478^{* *}$ & $0.585^{\circ *}$ & $0.716^{* *}$ & $0.707^{* *}$ & 1 & \\
\hline 8. Ipsicap Autoeficacia & $0.51^{* *}$ & $0.379^{* *}$ & $0.442^{* *}$ & $0.49^{* *}$ & $0.583^{* *}$ & $0.53^{* *}$ & $0.597^{* *}$ & \\
\hline 9. Ipsicap Total & $0.647^{* *}$ & $0.589^{* *}$ & $0.57^{* *}$ & $0.66^{\circ *}$ & $0.886^{* *}$ & $0.87^{* *}$ & $0.892^{* *}$ & $0.75^{* *}$ \\
\hline
\end{tabular}

De igual manera se procedió con la validez divergente. Con la tercera muestra, compuesta por 105 empleados de un colegio privado de la ciudad de Bogotá, se realizó la aplicación del Ipsicap-24 y el GHQ-28. Esta última prueba indaga sobre aspectos de salud física y mental, tales como: síntomas psicosomáticos, ansiedad y problemas de sueño, depresión y disfunción social. La evidencia ha demostrado que estos problemas de salud correlacionan de forma inversa con el capital psicológico (Avey, Wernsing, \& Mhatre, 2011; Cassidy, McLaughlin, \& McDowell, 2014; Rahimnia, Mazidi, \& Mohammadzadeh, 2013; Liu et al., 2013; Meseguer-de Pedro et al., 2017).

En la matriz expuesta en la Tabla 7 se aprecia que todas las correlaciones obtenidas entre estas dos pruebas son inversas y significativas $(p<$ $0.01, p<0.05)$, esto último excepto para el caso de la correlación entre esperanza y disfunción social $(r=-0.121)$, y autoeficacia y depresión $(r$ $=-0.104)$. Entre los coeficientes significativos, el valor más alto $(r=-0.504)$ corresponde a la correlación entre el Ipsicap-24 total y el GHQ-28 total, mientras que el más bajo $(r=-0.21)$ se produjo en la bina disfunción social y resiliencia.

\section{Tabla 7}

Matriz de Correlaciones Ipsicap-24-GHQ-28

\begin{tabular}{|c|c|c|c|c|c|c|c|c|c|}
\hline Pruebas & 1 & 2 & 3 & 4 & 5 & 6 & 7 & 8 & 9 \\
\hline 1. GHQ Psicosomáticos & & & & & & & & & \\
\hline 2. GHQ Ansiedad & $0.71^{* *}$ & & & & & & & & \\
\hline $\begin{array}{l}\text { 3. GHQ Disfunción social } \\
\text { 4. GHQ Depresión }\end{array}$ & $\begin{array}{l}0.478^{* *} \\
0.257^{*}\end{array}$ & $\begin{array}{c}0.508^{* *} \\
0.48^{*}\end{array}$ & $0.373^{* *}$ & & & & & & \\
\hline 5. GHQ Total & $0.845^{*}$ & $0.911^{*}$ & $0.721^{*}$ & $0.592^{* *}$ & & & & & \\
\hline ap $\mathrm{E}$ & $-0.263^{* 4}$ & $-0.358^{* 4}$ & -0.121 & $-0.488^{* \prime}$ & $-0.377^{*}$ & & & & \\
\hline apc $c$ & $-0.404^{*}$ & $-0.428^{* \prime}$ & $-0.276^{* *}$ & $-0.333^{* *}$ & $-0.467^{* *}$ & $0.515^{* *}$ & & & \\
\hline $\mathrm{R}$ & $-0.407^{* *}$ & $-0.333^{* *}$ & $-0.21^{*}$ & $-0.281^{* *}$ & $-0.402^{* *}$ & $0.516^{* *}$ & $0.597^{7 *}$ & & \\
\hline & $346^{*}$ & $-0.228^{\circ}$ & $-0.248^{*}$ & -00.104 & $-0.309^{* *}$ & & $0.445^{* x}$ & & \\
\hline 10. Ips & $-0.449^{* *}$ & $-0.443^{* *}$ & $-0.266^{* *}$ & $-0.413^{* *}$ & $-0.504^{* *}$ & $0.794^{* *}$ & $0.838^{* *}$ & $0.823^{* *}$ & \\
\hline
\end{tabular}

En conclusión, el Ipsicap-24 quedó conformado por cuatro escalas y 24 ítems (Tabla 4), distribuidos así: esperanza (7 ítems), optimismo (7 ítems), resiliencia (6 ítems) y autoeficacia (4 ítems). La distribución de ítems por escala se exhibe en la Tabla 8 . 
Tabla 8

Distribución de items por escala del Ipsicap-24

\begin{tabular}{llc}
\hline \multicolumn{1}{c}{ Escala } & \multicolumn{1}{c}{ N. $^{\text {. }}$ Ítem } & Total \\
\hline Esperanza & $1,2,3,4,5,6,7$ & 7 \\
Optimismo & $8,9,10,11,12,13,14$ & 7 \\
Resiliencia & $15,16,17,18,19,20$ & 6 \\
Autoeficacia & $21,22,23,24$ & 4 \\
\hline
\end{tabular}

\section{Discusión}

El objetivo del presente estudio se confirma porque el análisis de las propiedades psicométricas del Ipsicap-24 demuestra que es un instrumento válido y confiable. La consistencia interna de la prueba presenta correlaciones entre las binas formadas por las cuatro escalas constitutivas del Ipsicap-24 (Tablas 6 y 7), que son directas y significativas $(p<0.01)$, pero exhiben valores medios, lo que implica ausencia de multicolinealidad y refuerza la noción de que forman parte de un mismo constructo (Omar et al., 2014; Meseguer-de Pedro et al., 2017). En esta misma dirección, los resultados obtenidos mediante ecuaciones estructurales con el Ipsicap-24 (Figura 1) son coherentes con investigaciones que confirman al capital psicológico como constructo de segundo orden (Luthans et al., 2007; Avey, Reichard et al., 2011; Delgado-Abella \& Castañeda Zapata, 2011 \& Liu et al., 2013).

Con respecto a la validez de constructo, inicialmente se realizó un AFE (Tabla 4) que resultó de gran utilidad para depurar el Ipsicap original de 36 ítems, pues permitió observar que los ítems inversos que contenía este instrumento no miden directamente el constructo positivo, y por tal razón fueron eliminados. Por ejemplo, se pudo apreciar que un ítem como "He tenido que renunciar a trabajos donde constantemente había cambios" no representa una medida inversa de resiliencia, sino de otro constructo no identificado, probablemente adaptación al cambio, o más específicamente, inadaptación y/ o intención de cambiar de trabajo. Es pertinente recordar aquí que una característica distintiva de la resiliencia es la habilidad para adaptarse a los cambios (Lester, Vogelgesang, \& Saboe, 2018;
Soler-Sánchez, Fernández-Valera, \& Meseguerde Pedro, 2016; Robertson, Cooper, Sarkar, \& Curran, 2015; Rees, Breen, Cusack, \& Hegney, 2015; García-Día, DiNapoli, GarcíaOna, Jakubowski, \& O'flaherty, 2013). Este hallazgo confirma la postura de Peterson y Chang (2003), quienes, a partir de sus investigaciones sobre optimismo y pesimismo, afirman que constructos opuestos positivos y negativos no constituyen extremos de un mismo continuo; por el contrario, son bastante divergentes y poseen diferentes antecedentes, dimensiones $y$ resultados.

Otro aspecto para destacar en el presente estudio es la amplia y variada muestra de trabajadores colombianos pertenecientes a tres organizaciones distintas $(N=1517, N=428$ y $N=105)$, provenientes de diferentes sectores sociodemográficos y económicos, que permiten asegurar cierta heterogeneidad que puede actuar en favor de la consistencia interna de la prueba (Bandalos, 2018), al igual que el tamaño muestral grande (Abdelmoula, Chakroun, \& Akrout, 2015). Con respecto a las medidas de fiabilidad, los coeficientes Alfa de Cronbach para optimismo $(\alpha=0.78)$ y autoeficacia $(\alpha$ $=0.71)$ podrían considerarse adecuados, como lo sostienen Nunnally y Bernstein (1994); este mismo coeficiente para las escalas de esperanza $(\alpha$ $=0.84)$ y resiliencia $(\alpha=0.83)$ resultaría óptimo de acuerdo con Tavakol y Dennick (2011), quienes afirman que un alto valor Alfa (>0.9) podría sugerir redundancias y llegar a indicar que el tamaño de la prueba debería ser menor.

Además, los resultados obtenidos indican que el capital psicológico, evaluado mediante el Ipsicap-24, presenta correlaciones significativas con otros instrumentos de medida como son el UWES y el GHQ-28. Por una parte, el Ipsicap (Tabla 6) se relaciona de forma directa y significativa $(p<0.01)$ con el constructo engagement y sus tres componentes: vigor, absorción y dedicación, medidos a través del UWES. Esto aporta evidencia de validez convergente para el Ipsicap-24. Este hallazgo está en consonancia con varias investigaciones: Simons y Buitendach (2013) reportaron relaciones positivas y significativas 
entre capital psicológico (PCQ), engagement (UWES) y compromiso laboral (Organisational Commitment Questionnaire, OCQ); De Waal y Pienaar (2013), con un estudio longitudinal, probaron que el engagement (UWES) precede al capital psicológico (medidas independientes para cada componente); y Görgens-Ekermans y Herbert (2013), en un estudio dirigido a probar la validez interna y externa del PCQ-24, también hallaron correlaciones directas significativas entre capital psicológico y engagement. En Corea, Paek et al. (2015) sugieren que el engagement (UWES-9) actúa como mediador parcial sobre el efecto del capital psicológico (PCQ-24) sobre la satisfacción en el trabajo y el compromiso organizacional afectivo, estas dos últimas variables fueron medidas con ítems provenientes de varias escalas. En una muestra de la industria hotelera, Karatepe y Karadas (2015) encontraron que los empleados con alto capital psicológico (PCQ-24) también mostraban altos niveles de engagement (UWES); Lorenz et al. (2016) aportan información sobre correlaciones positivas y significativas entre el capital psicológico (CPC-12) y engagement (UWES). Por último, Li et al. (2018), en empresas de alta tecnología, informan que el capital psicológico (PCQ-24) predice positivamente el engagement de los empleados (UWES). Por otra parte, adicionalmente, en este estudio también se encontró evidencia de validez divergente para el Ipsicap-24 (Tabla 7). Se obtuvieron correlaciones inversas y significativas entre esta prueba y el GHQ-28, este último es un cuestionario de autoaplicación sobre síntomas psicosomáticos, ansiedad y problemas de sueño, depresión y disfunción social. La literatura confirma este hallazgo en varias investigaciones, como Avey, Wernsing et al. (2011), quienes presentaron que el capital psicológico (PCQ-24) correlaciona negativamente con estrés y ansiedad, medidas a través de Depression, Anxiety, and Stress Scale. Por su parte, Cassidy et al. (2014), usando el PCQ-24, el GHQ-12 y The Perceived Stress Scale (PSS), encontraron correlaciones negativas significativas entre cada uno de los cuatro componentes del capital psicológico y las medidas de salud mental negativa y estrés percibido. De igual manera, Rahimnia et al. (2013) informan sobre correlaciones negativas significativas entre capital psicológico (PCQ-24) y las medidas proporcionadas por Depression, Anxiety, and Stress Scale. Liu et al. (2013) también comunican una asociación significativa entre capital psicológico (PCQ-24) y síntomas depresivos (Center for Epidemiologic Studies Depression Scale, CES-D). Como parte del proceso de validación del OREA, Meseguerde Pedro et al. (2017) reportaron correlaciones negativas entre capital psicológico y las medidas arrojadas por el GHQ-12 sobre síntomas psicosomáticos.

El presente estudio nos deja otras importantes preguntas de investigación. Por un lado, las correlaciones entre esperanza y disfunción social $(r=-0.121)$, y autoeficacia y depresión $(r$ $=-0,104)$ no son significativas; este resultado no coincide con lo reportado en el párrafo anterior, razón por la cual se llama la atención sobre la necesidad de generar investigación que ayude a explicar esta situación. Por otro lado, también surge la necesidad de ahondar sobre la naturaleza diferencial de los constructos positivos y sus opuestos negativos, siguiendo la línea de las investigaciones sobre optimismo/pesimismo (Peterson \& Chang, 2003) y esperanza/desesperanza (Huen, Ip, Ho, \& Yip, 2015), que los asumen, en cada caso, como constructos diferentes pero relacionados.

Adicionalmente, como futuras investigaciones, sería conveniente aplicar el Ipsicap-24 en otros países, y así ver cómo se podrían generalizar los resultados. Por último, sería interesante utilizar otras metodologías, como la multinivel, que nos permitiera analizar estas variables desde la óptica individual, grupal u organizacional. También se recomienda hacer trabajos longitudinales con el fin de ver la influencia de causalidad a través del tiempo, por una parte, observando las dimensiones de la variable de capital psicológico y, por otra parte, observando la relación en el tiempo entre capital psicológico y las otras dos variables, engagement y percepción de salud.

Entre las limitaciones de la presente investigación se encuentra que el Ipsicap-24 es 
una medida de autoreporte, y las tres muestras están conformadas por trabajadores colombianos, por lo que se recomienda trabajar posteriormente con otros datos como: medidas fisiológicas, rendimiento con datos objetivos, bajas laborales, absentismo, etc., y también con muestras de otros países para aumentar la posibilidad de generalización de sus resultados y el desarrollo de estrategias de medición del capital psicológico basadas en la evidencia.

En conclusión, los resultados obtenidos con el Ipsicap-24 permiten confirmar la validación de este instrumento en población colombiana y se convierte en una herramienta de evaluación del "capital psicológico" con adecuadas propiedades psicométricas para ser utilizada en investigación y en procesos de análisis de los trabajadores para el desarrollo humano en el mundo del laboral.

\section{Referencias}

Abdelmoula, M., Chakroun, W., \& Akrout, F. (2015). The effect of sample size and the number of items on reliability coefficients: alpha and rhô: a meta-analysis. International Journal of Numerical Methods and Applications, 13(1), 1-20. http://doi.org /10.17654/IJNMAMar2015_001_020

Alessandri, G., Borgogni, L., Consiglio, C., \& Mitidieri, G. (2015). Psychometric properties of the italian version of the psychological capital questionnaire. International Journal of Selection and Assessment, 23(2), 149-159. https://doi.org/ 10.1111/ijsa.12103

Antunes, A., Caetano, A., \& Pina e Cunha, M. (2017). Reliability and construct validity of the Portuguese version of the Psychological Capital Questionnaire. Psychological reports, 120(3), 520-536. https://doi.org/10.1177/0 033294116686742

Avey, J., Luthans, F., \& Youssef, C. (2010). The additive value of positive psychological capital in predicting work attitudes and behaviors. Journal of Management, 36(2), 430-452. http://doi.org/10.1177/01492063 08329961
Avey, J., Reichard, R., Luthans, F., \& Mhatre, K. (2011). Meta-analysis of the impact of positive psychological capital on employee attitudes, behaviors, and performance. Human Resource Development Quarterly, $22(2), 127-152$. https://doi.org/10.1002/hrd q. 20070

Avey, J., Wernsing, T., \& Mhatre, K. (2011). A longitudinal analysis of positive psychological constructs and emotions on stress, anxiety, and well-being. Journal of Leadership and Organizational Studies, 18(2), 216-228. http://doi.org/10.1177/15480518 10397368

Azanza, G., Domínguez, Á., Moriano, J., \& Molero, F. (2014). Capital psicológico positivo. Validación del cuestionario PCQ en España. Anales De Psicología, 30(1), 294-301. http://doi.org/10.6018/analesps.3 0.1 .153631

Bandalos, D. (2018). Measurement theory and applications for the social sciences. Nueva York, NY: Guilford Publications.

Cassidy, T., McLaughlin, M., \& McDowell, E. (2014). Bullying and health at work: The mediating roles of psychological capital and social support. Work and Stress, 28(3), 255-269. https://doi.org/10.1080/02678373 .2014 .927020

Clapp-Smith, R., Vogelgesang, G., \& Avey, J. (2009). Authentic leadership and positive psychological capital: The mediating role of trust at the group level of analysis. Journal of Leadership Eु Organizational Studies, 15(3), 227-240. https://doi.org/10.1177/15480518 08326596

Cortina, J. M. (1993). What is coefficient alpha? An examination of theory and applications. Journal of applied psychology, 78(1), 98-104. https://doi.org/10.1037/0021-9010.78.1.98

Dawkins, S., Martin, A., Scott, J., \& Sanderson, K. (2013). Building on the positives: A psychometric review and critical analysis of the construct of psychological capital. Journal of Occupational and Organizational Psychology, 86(3), 348-370. http://doi.org/1 0.1111/joop.12007 
De Waal, J., \& Pienaar, J. (2013). Towards understanding causality between work engagement and psychological capital. SA Journal of Industrial Psychology, 39(2), 1-10, 433-452. https://doi.org/10.4102/sajip.v39i 2.1113

Delgado-Abella, L. (2013). La evaluación del capital psicológico en las organizaciones. En L. Delgado-Abella \& M. VanegasGarcía (Eds.), Psicología Organizacional: Perspectivas y Avances (pp. 21-39). Bogotá: Ecoe Ediciones.

Delgado-Abella, L., \& Castañeda Zapata, D. (2011). Relación entre capital psicológico y la conducta de compartir conocimiento en el contexto del aprendizaje organizacional. Acta Colombiana de Psicología, 14(1), 61-70. Recuperado de https://dialnet.unirioja.es/se rvlet/articulo? codigo $=5786578$

Delgado-Abella, L., Devia, L., \& Martínez, M. (2018). Diseño y validación mediante la teoría de respuesta al ítem del instrumento para evaluar capital psicológico en las organizaciones Ipsicap. En B. Avendaño, N. Ayala, S. Camelo, L. Delgado-Abella, J. Moreno, V. Sánchez \& N. Vargas (Eds.), Investigación en Psicología: Aplicaciones e Intervenciones (pp. 85-100). Bogotá: Universidad Católica de Colombia.

Djourova, N., Rodríguez, I., \& Lorente-Prieto, L. (2019). Validation of a Modified Version of the Psychological Capital Questionnaire (PCQ12) in Spain. Revista Interamericana de Psicología Ocupacional, 37(2), 93-106. http: //doi.org/10.21772/ripo.v37n2a02

Dollwet, M., \& Reichard, R. (2014). Assessing cross-cultural skills: Validation of a new measure of cross-cultural psychological capital. International Journal of Human Resource Management, 25(12), 1669-1696. https://doi.org/10.1080/095851 92.2013.845239

Escobedo Portillo, M., Hernández Gómez, J., Estebané Ortega, V., \& Martínez Moreno, G. (2016). Modelos de ecuaciones estructurales: características, fases, construcción, aplicación y resultados.
Ciencia $\mathbb{E}$ trabajo, 18(55), 16-22. http://doi .org/10.4067/S0718-24492016000100004

García-Día, M., DiNapoli, J., García-Ona, L., Jakubowski, R., \& O'flaherty, D. (2013). Concept analysis: resilience. Archives of psychiatric nursing, 27(6), 264-270. http://d oi.org/10.1016/j.apnu.2013.07.003

Goldberg, D., \& Hillier, V. (1979). A scaled version of the General Health Questionnaire. Psychological medicine, 9(1), 139-145. https://doi.org/10.1017/S0033291 700021644

Görgens-Ekermans, G., \& Herbert, M. (2013). Psychological capital: Internal and external validity of the psychological capital questionnaire (PCQ-24) on a South African sample. SA Journal of Industrial Psychology, 39(2), 1-12. https://doi.org/10.4 102/sajip.v39i2.1131

Harms, P., \& Luthans, F. (2012). Measuring implicit psychological constructs in organizational behavior: An example using psychological capital. Journal of Organizational Behavior, 33(4), 589-594. ht tps://doi.org/10.1002/job.1785

Harms, P. D., Vanhove, A., \& Luthans, F. (2017). Positive projections and health: An initial validation of the implicit psychological capital health measure. Applied Psychology, 66(1), 78-102. https://doi.org/10.1111/apps .12077

Heled, E., Somech, A., \& Waters, L. (2016). Psychological capital as a team phenomenon: Mediating the relationship between learning climate and outcomes at the individual and team levels. Journal of Positive Psychology, 11 (3), 303-314. https://d oi.org/10.1080/17439760.2015.1058971

Huen, J. M. Y., Ip, B. Y. T., Ho, S. M. Y., \& Yip, P. S. F. (2015). Hope and Hopelessness: The Role of Hope in Buffering the Impact of Hopelessness on Suicidal Ideation. Plos One, 10(6), e0130073. https://doi.org/10.1 371/journal.pone.0130073

Kamei, H., Ferreira, M., Valentini, F., Peres, M., Kamei, P., \& Damásio, B. (2018). Psychological Capital Questionnaire-Short Version (PCQ-12): evidence of Validity 
of The Brazilian Version. Psico-Usf, 23(2), 203-214. http://doi.org/10.1590/1413-8271 2018230202

Karatepe, O., \& Karadas, G. (2015). Do psychological capital and work engagement foster frontline employees' satisfaction? A study in the hotel industry. International Journal of Contemporary Hospitality Management, 27(6), 1254-1278. https://doi .org/10.1108/IJCHM-01-2014-0028

León-Pérez, J., Antino, M., \& León-Rubio, J. (2017). Adaptation of the short version of the Psychological Capital Questionnaire (PCQ-12) into Spanish/ Adaptación al español de la versión reducida del Cuestionario de Capital Psicológico (PCQ-12). Revista de Psicología Social, 32(1), 196-213. https://doi.org/10.1 080/02134748.2016.1248024

Lester, P., Vogelgesang, G., \& Saboe, K. (2018). Resilience within the workplace. Organizational Dynamics, 47(4), 201-208. ht tps://doi.org/10.1016/j.orgdyn.2018.01.005

Li, Y., Castaño, G., \& Li, Y. (2018). Linking leadership styles to work engagement: The role of psychological capital among Chinese knowledge workers. Chinese Management Studies, 12 (2), 433-452. https://doi.org/10.1 108/CMS-04-2017-0108

Liu, L., Hu, S., Wang, L., Sui, G., \& Ma, L. (2013). Positive resources for combating depressive symptoms among chinese male correctional officers: Perceived organizational support and psychological capital. BMC Psychiatry, 13(89), 1-9. https: //doi.org/10.1186/1471-244X-13-89

López-Núñez, M., de Jesús, S., Viseu, J., \& Santana-Cárdenas, S. (2018). Capital Psicológico de los Trabajadores en España. Análisis Factorial Confirmatorio del PCQ-12. Revista Iberoamericana de Diagnóstico y Evaluación-e Avaliação Psicológica, 3(48), 67-79. https://doi.org/10. 21865/RIDEP48.3.06

Lorenz, T., Beer, C., Pütz, J., \& Heinitz, K. (2016). Measuring psychological capital: Construction and validation of the compound PsyCap scale (CPC-12). Plos
One, 11(4), 1-17. https://doi.org/10.1371/jo urnal.pone.0152892

Lupşa, D., \& Vîrgă, D. (2018). Psychological Capital Questionnaire (PCQ): Analysis of the Romanian Adaptation and Validation. Human Resources Psychology/ Psihologia Resurselor Umane, 16(1), 27-39.

Luthans, F., Avolio, B., Avey, J., \& Norman, S. (2007). Positive psychological capital: Measurement and relationship with performance and satisfaction. Personnel Psychology, 60(3), 541-572. https://doi.org/ 10.1111/j.1744-6570.2007.00083.x

Luthans, F., \& Youssef-Morgan, C. (2017). Psychological capital: An Evidence-Based positive approach. Annual Review of Organizational Psychology and Organizational Behavior, 4, 339-366. https://doi.org/10.114 6/annurev-orgpsych-032516-113324

Luthans, F., Youssef, C., \& Avolio, B. (2007). Psychological capital: investing and developing positive organizational behavior. En D., Nelson, Eु C., Cooper, Positive organizational behavior. Chennai: SAGE Publications.

Meseguer-de Pedro, M., Soler-Sánchez, M., Fernández-Valera, M., \& García-Izquierdo, M. (2017). Measurement of psychological capital in spanish workers: OREA questionnaire. Anales De Psicología, 33(3), 714-721.

Newman, A., Ucbasaran, D., Zhu, F., \& Hirst, G. (2014). Psychological capital: A review and synthesis. Journal of Organizational Behavior, 35(1), 120-138. https://doi.org/10.1002/job .1916

Nolzen, N. (2018). The concept of psychological capital: a comprehensive review. Management Review Quarterly, 68(3), 237-277. https://doi.org/10.1007/s11 301-018-0138-6

Nunnally, J., \& Bernstein, L. (1994). Psychometric Theory. Nueva York, NY: McGraw-Hill.

Omar, A., Salessi, S., \& Urteaga, F. (2014). Diseño y validación de la escala Cappsi para medir capital psicológico. Liberabit, 20(2), 315-323. Recuperado de http://hdl.handle. net/11336/29909 
Ospina, C., \& Delgado-Abella, L. (2014). Adaptación del Cuestionario Utrecht Work Engagament (UWES) a la población colombiana. Bogotá: Universidad Católica de Colombia.

Paek, S., Schuckert, M., Kim, T., \& Lee, G. (2015). Why is hospitality employees' psychological capital important? The effects of psychological capital on work engagement and employee morale. International Journal of Hospitality Management, 50, 9-26. https://doi.org/10.10 16/j.ijhm.2015.07.001

Peterson, C., \& Chang, E. (2003). Optimism and flourishing. En C. Keyes \& J. Haidt (Eds.), Flourishing: Positive psychology and the life well-lived (pp. 55-79). Washington, DC: Asociación Estadounidense de Psicología. h ttp://doi.org/10.1037/10594-000

Rahimnia, F., Mazidi, A., \& Mohammadzadeh, Z. (2013). Emotional mediators of psychological capital on well-being: The role of stress, anxiety, and depression. Management Science Letters, 3(3), 913-926. https://doi.org/10.5267/j.msl.2013.01.029

Rees, C., Breen, L., Cusack, L., \& Hegney, D. (2015). Understanding individual resilience in the workplace: the international collaboration of workforce resilience model. Frontiers in psychology, 6, 1-7. https://doi.org/10.3389/fpsyg.2015.000 73

Rego, A., Owens, B., Yam, K., Bluhm, D., Cunha, M., Silard, A., ...Liu, W. (2019). Leader humility and team performance: Exploring the mediating mechanisms of team PsyCap and task allocation effectiveness. Journal of Management, 45(3), 1009-1033. https://doi .org/10.1177/0149206316688941

Ricker, K. (2006). Setting Cut-Scores: A Critical Review of the Angoff and Modified Angoff Methods. The Alberta Journal of Educational Research, 52 (1), 53-64. Recuperado de https://www.search-proquest-com.ezpro xy.unbosque.edu.co/central/docview/2286 39019/fulltextPDF/5E918879CB7D414BP $\mathrm{Q} / 1$ ?accountid=41311
Robertson, I., Cooper, C., Sarkar, M., \& Curran, T. (2015). Resilience training in the workplace from 2003 to 2014: A systematic review. Journal of Occupational and Organizational Psychology, 88(3), 533-562. h ttps://doi.org/10.1111/joop.12120

Ruiz, M., Pardo, A., \& San Martín, R. (2010). Modelos de ecuaciones estructurales. Papeles del psicólogo, 31(1), 34-45. Recuperado de http://www.papelesd elpsicologo.es/pdf/1792.pdf

Santana-Cárdenas, S., Viseu, J., López-Núñez, M., \& Jesus, S. (2018). Evidencias psicométricas sobre la validez y fiabilidad del cuestionario de capital psicológico (PCQ-12) en población mexicana. Anales de Psicología/Annals of Psychology, 34(3), 562-570. http://doi.org/10.6018/analesps.3 4.3.319211

Sapyaprapa, S., Tuicomepee, A., \& Watakakosol, R. (2013). Validation of psychological capital questionnaire in Thai employees. Proceedings of the Asian Conference on Psychology and the Behavioral Sciences. Tokio, Japón.

Schaufeli, W., \& Bakker, A. (2003). Job demands, job resources, and their relationship with burnout and engagement: a multi-sample study. Journal of Organizational Behavior, 25(3), 293-315. https://doi.org/10.1002/job .248

Simons, J., \& Buitendach, J. (2013). Psychological capital, work engagement and organisational commitment amongst call centre employees in South Africa. SA Journal of Industrial Psychology, 39(2), 1-12. https://doi.org/10.4102/sajip.v39i2.1071

Soler-Sánchez, M., Fernández-Valera, M., \& Meseguer-de Pedro, M. (2016). El papel moderador de la resiliencia entre situaciones de demandas de rol laboral y de malestar autopercibido. Revista Mexicana de Psicología, 33(1), 61-70. Recuperado de http://www.redalyc.org/articulo.oa?id= 243056043007

Somech, A., \& Khotaba, S. (2017). An integrative model for understanding team organizational citizenship behavior: 
Its antecedents and consequences for educational teams. Journal of Educational Administration, 55(6), 671-685. https://doi. org/10.1108/JEA-09-2016-0104

Srivastava, U., \& Maurya, V. (2017). Organizational and Individual Level Antecedents of Psychological Capital and its Associated Outcomes: Development of a Conceptual Framework. Management and Labour Studies, 42(3), 205-236. https://doi. org/10.1177/0258042X17718739

Tang, W., Cui, Y., \& Babenko, O. (2014). Internal consistency: Do we really know what it is and how to assess it. Journal of Psychology and Behavioral Science, 2(2), 205-220. Recuperado de http://jpbsnet.com/journals /jpbs/Vol_2_No_2_June_2014/13.pdf

Tavakol, M., \& Dennick, R. (2011). Making sense of cronbach's alpha. International Journal of Medical Education, 2, 53-55. https://www.ijme.net/archive/2/cr onbachs-alpha/

Walumbwa, F., Luthans, F., Avey, J., \& Oke, A. (2011). Authentically leading groups: The mediating role of collective psychological capital and trust. Journal of Organizational Behavior, 32, 4-24. http://dx.doi.org/10.100 2/job.653

\section{Notas}

* Artículo de investigación. 